\title{
Duality and fluctuation relations for statistics of currents on cyclic graphs
}

\author{
Jie Ren ${ }^{1,2}$, V. Y. Chernyak ${ }^{3,4}$, and N. A. Sinitsyn ${ }^{4,5}$ \\ ${ }^{1}$ NUS Graduate School for Integrative Sciences and Engineering, Singapore 117456, Republic of Singapore \\ ${ }^{2}$ Department of Physics and Centre for Computational Science and Engineering, \\ National University of Singapore, Singapore 117546, Republic of Singapore \\ ${ }^{3}$ Department of Chemistry, Wayne State University, 5101 Cass Ave,Detroit, MI 48202 \\ ${ }^{4}$ Theoretical Division, Los Alamos National Laboratory, B258, Los Alamos, NM 87545 and \\ ${ }^{5}$ New Mexico Consortium, Los Alamos, NM 87544, USA
}

(Dated: June 7, 2018)

\begin{abstract}
We consider stochastic motion of a particle on a cyclic graph with arbitrarily periodic time dependent kinetic rates. We demonstrate duality relations for statistics of currents in this model and in its continuous version of a diffusion in one dimension. Our duality relations are valid beyond detailed balance constraints and lead to exact expressions that relate statistics of currents induced by dual driving protocols. We also show that previously known no-pumping theorems and some of the fluctuation relations, when they are applied to cyclic graphs or to one dimensional diffusion, are special consequences of our duality.

PACS numbers: 05.60.-k, 05.40.-a, 82.37.-j, 82.20.-w
\end{abstract}

\section{INTRODUCTION}

Dynamics of mesoscopic structures can often be modeled as a stochastic motion on a network [1-4]. Nodes of this network represent metastable states of a structure and links correspond to allowed transitions. Examples can be found among biological enzymes [5, 6], electronic circuits [7, 8] and heat pumps [9]. Typically, nanomechanical systems should be able to perform cyclic operations, which correspond to motion along the cycles in such networks [1, 2].

In this article, our main focus will be on stochastic particle motion in a cyclic graph. In such a graph, nodes are connected one by one, forming a closed chain, i.e. a cycle. Number of nodes of a cyclic graph is equal to the number of its links and each node has exactly two neighboring nodes to which it is connected. This graph is already a sufficiently general representation of the kinetics for many nanoscale structures. Understanding dynamics on such a graph is also a starting point for understanding more complicated systems. For example, arbitrary networks can be decomposed into fundamental cycles [1]. In such a graph, we will study counting statistics of current induced by time-dependent driving and by forces that explicitly break the time-reversal symmetry.

Our main result is that the Markovian kinetic on cyclic graphs with arbitrary time-dependent kinetic rates has exact duality for statistics of particle currents. This duality leads to new exact relations for statistics of currents in systems driven by time-dependent protocols. These relations are akin to known fluctuation theorems for currents [10] but they are applied to systems with arbitrary time dependence of all kinetic rates. In fact, for cyclic graphs, we show that known fluctuation relations, as well as other known exact results called no-pumping theorems, are special consequences of our duality relations.

\section{MASTER EQUATION AND ITS DUAL EQUATION ON A CYCLIC GRAPH}

Consider a cyclic graph with $N$ nodes and $N$ links. Links represent allowed transitions between nodes. We assume that all links can be traversed in both directions with some rates. Detailed balance is not assumed. Let $k_{i}^{+}$and $k_{i}^{-}$be the kinetic rates of transitions from state $i$ into, respectively, states $i+1$ and $i-1$, as shown in Fig. 1 (a). We will identify indices $i=0$ as $i=N$, and $i=N+1$ as $i=1$ if necessarily. The evolution of the probability vector $\boldsymbol{p}$ is given by the master equation:

$$
d_{t} \boldsymbol{p}=\hat{H} \boldsymbol{p}
$$

where we will call $\hat{H}$ the master operator. Let $\langle j|$ and $|i\rangle$ be the bra- and ket- $N$-vectors with the only nonzero unit components at $j$-th and $i$-th positions respectively. In this basis, the operator $\hat{H}$ has components $H_{i \pm 1, i}=k_{i}^{ \pm}, H_{i, i}=-k_{i}^{+}-k_{i}^{-}$, and zero otherwise. The constraint of column-sum-zero on the master operator,

$$
\sum_{i=1}^{N} H_{i, j}=0
$$

implies the conservation of the probability on a graph, $\sum_{i=1}^{N} p_{i}=1$.

The master operator, $\hat{H}$, can be written as a product of two operators:

$$
\hat{H}=\hat{\mathcal{Q}} \hat{J}
$$

where

$$
\hat{\mathcal{Q}}|i\rangle \equiv|i-1\rangle-|i\rangle \quad \hat{J}|i\rangle \equiv k_{i}^{-}|i\rangle-k_{i}^{+}|i+1\rangle .
$$

We will need the following property, namely, with each master operator $\hat{H}$ on a cyclic graph, we can associate a dual master operator, given by [11]:

$$
\hat{H}^{\mathrm{d}}=\hat{\mathcal{Q}}^{\mathrm{T}} \hat{J}^{\mathrm{T}},
$$


where $\mathrm{T}$ is a transposition operation. In components, we have

$$
\hat{H}_{i, i}^{\mathrm{d}}=-k_{i}^{-}-k_{i-1}^{+}, \quad \hat{H}_{i+1, i}^{\mathrm{d}}=k_{i}^{-}, \quad \hat{H}_{i-1, i}^{\mathrm{d}}=k_{i-1}^{+} .
$$

The operator, $\hat{H}^{\mathrm{d}}$, satisfies the probability conservation condition Eq. (2) and hence it is a valid master operator describing stochastic particle motion on a cyclic graph with kinetic rates:

$$
k_{i}^{\mathrm{d}+}=k_{i}^{-}, \quad k_{i}^{\mathrm{d}-}=k_{i-1}^{+} .
$$

\section{STATISTICS OF CURRENTS ON A CYCLIC GRAPH}

Suppose that $J$ is a random variable, which we call the current, defined so that whenever the particle makes a jump (transition) in the clockwise direction we increase $J$ by unity $(J \rightarrow J+1)$, and whenever the particle makes a jump in the counter-clockwise direction we decrease $J$ by unity $(J \rightarrow J-1)$. Here we assume that all kinetic rates are time-dependent and change according to some periodic protocol with period $\tau$. Such a steadily driven system eventually enters a regime with periodically changing population probability vector, i.e. $\boldsymbol{p}(t+\tau)=\boldsymbol{p}(t)$. We also introduce the current per period of the driving protocol in the limit of large observation time, which is also called the conserved current,

$$
J^{c}=\lim _{n \rightarrow \infty} \frac{1}{n} \int_{0}^{n \tau} d t J(t),
$$

where $n$ is the number of cycles of the driving protocol. In general, the distribution of conserved current has an asymptotic form $P\left(\int_{0}^{n \tau} d t J(t)\right) \sim \exp \left(n \mathcal{S}\left(J^{c}\right)\right)$ in the limit of large $n$, or written as

$$
P\left(J^{c}\right) \sim e^{\mathcal{S}\left(J^{c}\right)},
$$

where $\mathcal{S}\left(J^{c}\right)$ is called the large deviation function (LDF) [12]. The cumulant generating function (CGF), $\mu_{\chi}$, generates cumulants of the conserved current and is defined through the relation $\left\langle\exp \left(\chi \int_{0}^{n \tau} d t J\right)\right\rangle \sim \exp \left(n \mu_{\chi}\right)$, or say:

$$
\left\langle e^{\chi J^{c}}\right\rangle \sim e^{\mu_{\chi}} .
$$

The $i$ th order derivative of $\mu_{\chi}$ with respect to the auxiliary counting parameter $\chi$, at $\chi=0$ gives the $i$ th order cumulant of the conversed current. Moreover, the CGF is connected with the LDF through the Legendre transform $\mu_{\chi}=$ $\operatorname{Max}_{J^{c}}\left[\chi J^{c}+\mathcal{S}\left(J^{c}\right)\right]$ [13]. Thus, once we find the CGF, we are able to obtain the LDF through the inverse Legendre transform so that the distribution of the conversed current can be reconstructed.

There is a well developed and reviewed approach to calculate $\mu_{\chi}$ for models of stochastic evolution [4, 8, 10, 14-16]. Let $P_{i, J}(t)$ be the probability that at time $t$ the particle will be on the $i$-th node and by this time the produced current will be $J$. Then the master equation for probabilities $P_{i, J}(t)$ reads

$$
\frac{d}{d t} P_{i, J}=-\left(k_{i}^{+}+k_{i}^{-}\right) P_{i, J}+k_{i+1}^{-} P_{i+1, J+1}+k_{i-1}^{+} P_{i-1, J-1} .
$$

Let $\boldsymbol{Z}=\boldsymbol{Z}(\chi, t)$ be the vector of generating functions with components $Z_{i}=\sum_{J=-\infty}^{\infty} P_{i, J}(t) e^{\chi J}$. Multiplying Eq. (11) by $e^{\chi^{J}}$ and summing over $J$, we find that the generating function $\boldsymbol{Z}$ satisfies the following equation:

$$
d_{t} \boldsymbol{Z}=\hat{H}_{\chi} \boldsymbol{Z}
$$

which we will call the twisted master equation. We will call $\hat{H}_{\chi}$ the twisted master operator. It has off-diagonal components given by $\left(\hat{H}_{\chi}\right)_{i \pm 1, i}=\hat{H}_{i \pm 1, i} e^{ \pm \chi}$. The term "twisted" is justified here because the operator, $\hat{H}_{\chi}$, can be obtained from the master operator, $\hat{H}$, in Eq. (1) by multiplying (twisting) its off-diagonal matrix elements, responsible for clockwise or counter-clockwise transitions, with auxiliary parameter $e^{\chi}$ or $e^{-\chi}$ respectively.

A formal solution of Eq. (12) can be written in the form

$$
\boldsymbol{Z}(\chi, \tau) \equiv e^{\mu_{\chi}}=\hat{U}_{\chi} \boldsymbol{p}(0), \quad \hat{U}_{\chi}=\hat{T} e^{\int_{0}^{\tau} \hat{H}_{\chi}(t)},
$$

where $U_{\chi}=U_{\chi}(\tau)$ is the corresponding evolution operator and $\boldsymbol{p}(0)$ is the vector of the initial population probabilities of the nodes. To obtain statistics of the conserved current, $J^{c}$, we note that in the limit of large observation time the information about the initial state vector, $\boldsymbol{p}(0)$, becomes irrelevant and the evolution of $\boldsymbol{Z}$ is dominated by the largest eigenvalue of the evolution operator, $U_{\chi}$. The logarithm of this eigenvalue gives the CGF of currents $\mu_{\chi}$, namely,

$$
\hat{U}_{\chi}\left|u_{\chi}\right\rangle=e^{\mu_{\chi}}\left|u_{\chi}\right\rangle,
$$

where $\left|u_{\chi}\right\rangle$ is the eigenstate of $\hat{U}_{\chi}$ that corresponds to the largest eigenvalue.

Similarly, for the dual master operator, $\hat{H}^{\mathrm{d}}$, we can build its twisted version, $\hat{H}_{\chi}^{\mathrm{d}}$, by multiplying its off-diagonal matrix elements, which are responsible for clockwise or counterclockwise transitions, with $e^{\chi}$ or $e^{-\chi}$, respectively. The analogous evolution operator and the CGF for the dual graph are given by

$$
\hat{U}_{\chi}^{\mathrm{d}}=\hat{T} e^{\int_{0}^{\tau} \hat{H}_{\chi}^{\mathrm{d}}(t)}, \quad \hat{U}_{\chi}^{\mathrm{d}}\left|u_{\chi}^{\mathrm{d}}\right\rangle=e^{\mu_{\chi}}\left|u_{\chi}^{\mathrm{d}}\right\rangle,
$$

with $\left|u_{\chi}^{\mathrm{d}}\right\rangle$ the eigenstate of $\hat{U}_{\chi}^{\mathrm{d}}$ corresponding to the largest eigenvalue.

\section{DUALITY IN TWISTED MASTER EQUATION}

The duality on the level of master operators has been discussed previously [11, 17]. Here we will show that a similar duality can be introduced on the level of twisted master operators, which describe statistics of currents in original and dual models.

Let us introduce two twisted operators in cyclic graphs with arbitrary driving, namely:

$$
\hat{\mathcal{Q}}_{\chi}|i\rangle \equiv|i-1\rangle-e^{\chi}|i\rangle, \quad \hat{J}_{\chi}|i\rangle \equiv e^{-\chi} k_{i}^{-}|i\rangle-k_{i}^{+}|i+1\rangle,
$$


(a)

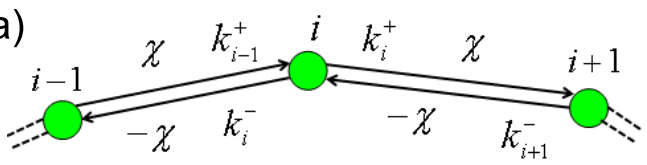

(b)

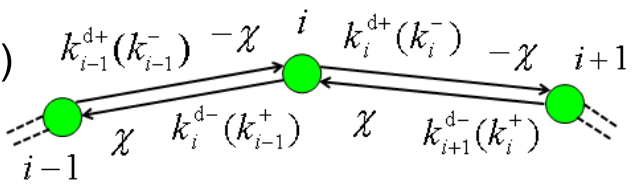

FIG. 1: Sketch maps for parameters of the twisted master operators on links connected to state $i$ for (a) original kinetic model and (b) its dual graph.

where the former is a constant operator while the latter contains the time-dependent transition rates. With these operators, two twisted master operators can be built, namely

$$
\hat{H}_{\chi}=\hat{\mathcal{Q}}_{\chi} \hat{J}_{\chi}, \quad \text { and } \quad \hat{H}_{-\chi}^{\mathrm{d}}=\hat{\mathcal{Q}}_{\chi}^{\mathrm{T}} \hat{J}_{\chi}^{\mathrm{T}} .
$$

One can check that $\hat{H}_{\chi}$ is a twisted master operator that corresponds to the evolution of the counting statistics in the original kinetic model, as seen in Eq. (12). While $\hat{H}_{-\chi}^{\mathrm{d}}$ is the twisted master operator that corresponds to the master operator $\hat{H}^{\mathrm{d}}$ of the dual model, however, with the counting parameter $\chi$ $(-\chi)$ replaced by $-\chi(\chi)$. The master operators in the original and the dual models, $\hat{H}_{\chi}$ and $\hat{H}_{-\chi}^{\mathrm{d}}$, are further connected with each other by the duality relation for their kinetic rates:

$$
k_{i}^{\mathrm{d}+}(t)=k_{i}^{-}(t), \quad k_{i}^{\mathrm{d}-}(t)=k_{i-1}^{+}(t),
$$

as illustrated in Fig. 1

Let us denote $\widetilde{H}_{-\chi} \equiv \hat{H}_{\chi}^{\mathrm{T}}=\hat{J}_{\chi}^{\mathrm{T}} \hat{\mathcal{Q}}_{\chi}^{\mathrm{T}}$. Since $\widetilde{H}_{-\chi}$ and $\hat{H}_{\chi}$ differ merely by a transposition, their evolution operators $\widetilde{U}_{-\chi}=\hat{T} \exp \left(\int_{0}^{\tau} d t \widetilde{H}_{-\chi}\right)$ and $\hat{U}_{\chi}=\hat{T} \exp \left(\int_{0}^{\tau} d t \hat{H}_{\chi}\right)$ have a "time-reversal" correspondence as a consequence of the time ordering operator $\hat{T}$, namely,

$$
\hat{U}_{\chi, \mathrm{F}}=\left(\widetilde{U}_{-\chi, \mathrm{B}}\right)^{\mathrm{T}} .
$$

Indexes "F" and "B" correspond to "forward" and "backward" protocols, such that the time-dependent parameters are related by $k_{i, \mathrm{~B}}(t)=k_{i, \mathrm{~F}}(\tau-t)$. (In what follows, we omit this index if the quantities at both sides of an equation belong to the same "forward" or "backward" protocols.) Similarly, let $\widetilde{H}_{\chi}^{\mathrm{d}} \equiv\left(\hat{H}_{-\chi}^{\mathrm{d}}\right)^{\mathrm{T}}=\hat{J}_{\chi} \hat{\mathcal{Q}}_{\chi}$ be the "time-reversal" counterpart of the dual twisted master operator, $\hat{H}_{-\chi}^{\mathrm{d}}$. Corresponding evolution operators, $\widetilde{U}_{\chi}^{\mathrm{d}}=\hat{T} \exp \left(\int_{0}^{\tau} d t \widetilde{H}_{\chi}^{\mathrm{d}}\right)$ and $\hat{U}_{-\chi}^{\mathrm{d}}=\hat{T} \exp \left(\int_{0}^{\tau} d t \hat{H}_{-\chi}^{\mathrm{d}}\right)$, satisfy the relation:

$$
\widetilde{U}_{\chi, \mathrm{F}}^{\mathrm{d}}=\left(\hat{U}_{-\chi, \mathrm{B}}^{\mathrm{d}}\right)^{\mathrm{T}} .
$$

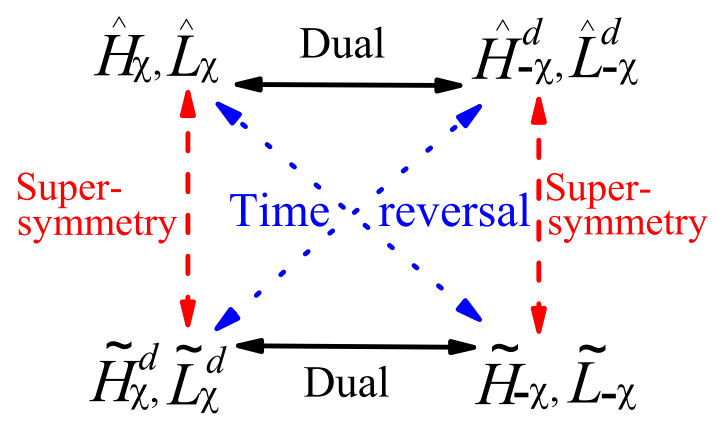

FIG. 2: The duality and hidden symmetry relations among the four twisted discrete operators as well as their continuous correspondences, the four twisted Fokker-Planck operators.

$\hat{H}_{\chi}$ and $\widetilde{H}_{\chi}^{\mathrm{d}}$ satisfy the obvious relations $\hat{H}_{\chi} \hat{\mathcal{Q}}_{\chi}=\hat{\mathcal{Q}}_{\chi} \widetilde{H}_{\chi}^{\mathrm{d}}$ and $\hat{H}_{-\chi}^{\mathrm{d}} \hat{\mathcal{Q}}_{\chi}^{\mathrm{T}}=\hat{\mathcal{Q}}_{\chi}^{\mathrm{T}} \widetilde{H}_{-\chi}$, and can be regarded as the supersymmetric counterparts to each other [11,21], as well as $\hat{H}_{-\chi}^{\mathrm{d}}$ and $\widetilde{H}_{-\chi}$. The relations among the four twisted operators are summarized in Fig. 2

These four twisted discrete operators are isospectral for arbitrary time-dependent protocols. This follows from the fact that the operator $\hat{\mathcal{Q}}_{\chi}$ is time-independent, and if $\left|\widetilde{u}_{\chi}^{\mathrm{d}}(t)\right\rangle$ satisfies the equation $d_{t}\left|\widetilde{u}_{\chi}^{\mathrm{d}}(t)\right\rangle=\widetilde{H}_{\chi}^{\mathrm{d}}\left|\widetilde{u}_{\chi}(t)\right\rangle$ then the vector $\left|u_{\chi}(t)\right\rangle=\hat{\mathcal{Q}}_{\chi}\left|\widetilde{u}_{\chi}^{\mathrm{d}}(t)\right\rangle$ immediately satisfies the original twisted master equation $d_{t}\left|u_{\chi}(t)\right\rangle=\hat{H}_{\chi}\left|u_{\chi}(t)\right\rangle$. Since solutions of evolution equations with $\widetilde{H}_{\chi}^{\mathrm{d}}(t)$ and $\hat{H}_{\chi}(t)$ coincide up to multiplication by a constant matrix, the eigenvalues of the corresponding evolution operators $\widetilde{U}_{\chi}^{\mathrm{d}}(t)$ and $\hat{U}_{\chi}(t)$, are the same. Further, by noticing that the transposition only reverses the time ordering, while keeps the eigenvalues unchanged, the matrices $\hat{U}_{\chi, \mathrm{F}}, \hat{U}_{-\chi, \mathrm{B}}^{\mathrm{d}}, \widetilde{U}_{\chi, \mathrm{F}}$ and $\widetilde{U}_{-\chi, \mathrm{B}}^{\mathrm{d}}$ share the identical eigen-spectrum.

As a consequence, evolution operators for counting statistics in the original model and its dual counterpart with a timereversed driving protocol have the same largest eigenvalues and, hence, the same CGFs. We are now in a position to formulate our findings in the form of the duality relation for currents, stating that:

For any driving protocol on a cyclic graph with periodically time-dependent rates, $k_{i}^{ \pm}(t)$, there is a dual protocol, defined as

$$
k_{i, \mathrm{~B}}^{\mathrm{d}+}(t)=k_{i, \mathrm{~F}}^{-}(\tau-t), \quad k_{i, \mathrm{~B}}^{\mathrm{d}-}(t)=k_{i-1, \mathrm{~F}}^{+}(\tau-t),
$$

with $i=1, \ldots N$. The cumulant generating functions for the original and the dual protocols satisfy the relation:

$$
\mu_{\chi, \mathrm{F}}=\mu_{-\chi, \mathrm{B}}^{\mathrm{d}} .
$$

After the inverse Legendre transformation, Eq. (22) leads to the following relation between $L D$-functions and probability distributions for conserved currents generated by original and dual protocols,

$$
\mathcal{S}_{\mathrm{F}}\left(J^{c}\right)=\mathcal{S}_{\mathrm{B}}^{\mathrm{d}}\left(-J^{c}\right), \quad P_{\mathrm{F}}\left(J^{c}\right)=P_{\mathrm{B}}^{\mathrm{d}}\left(-J^{c}\right) .
$$


Eq. (23) can be called a fluctuation relation in a sense that it describes the full statistics of currents in arbitrarily driven system. Eq. (23) is the main result of this article. It shows that exact fluctuation relations can follow from duality rather than from relations among work, free energy and entropy.

\section{DUALITY AND PREVIOUSLY KNOWN EXACT RESULTS}

Beyond the fluctuation relation (23), the duality provides us with a method to generate new fluctuation relations from already known ones. Here we demonstrate how some of the nontrivial known results could be derived using our duality transformation.

\section{A. No-Pumping Theorems}

When the discrete kinetic model corresponds to a system with strong coupling to thermal environment at inverse temperature $\beta$, its kinetic rates, following the Arrhenius law, may be parameterized as $k_{i-1}^{+}=k e^{\beta\left(E_{i-1}-W_{i}\right)}$ and $k_{i}^{-}=$ $k e^{\beta\left(E_{i}-W_{i}\right)}$ with some parameters $k, W_{i}$ and $E_{i}$, where $E_{i}$ corresponds to the size of the energy well of node $i$, and $W_{i}$ corresponds to the size of the barrier separating nodes $i$ and $i-1$. In this parametrization, recalling the relation Eq. (21), original and dual protocols are related as follows,

$$
E_{i, \mathrm{~B}}^{\mathrm{d}}(t)=-W_{i, \mathrm{~F}}(\tau-t), \quad W_{i+1, \mathrm{~B}}^{\mathrm{d}}(t)=-E_{i, \mathrm{~F}}(\tau-t),
$$

i.e., the dual model is different from the original one by exchange of energy wells $\left\{E_{i}\right\}$ with inverted barriers $\left\{W_{i}\right\}$. The duality relation means, in particular, that if we prove some general property for a model in which only barriers are driven at arbitrary values then automatically the same property follows for any model with the same geometry in which barriers are fixed but energies are driven.

We can use this fact. For example, if we drive only barriers $W_{i}$, then state probabilities remain constant, which is trivial to check, and hence currents are zero on average. The dual counterpart of this trivial result is the no-pumping theorem [18, 19] stating that periodic driving of node energies $E_{i}$ cannot induce a current on average. By analogy, breaking the detailed balance by a time-independent non-conserving force one can arrive at the generalized no-pumping theorem [20].

\section{B. Fluctuation Relations for Currents}

When the detailed balance condition is broken, kinetic rates on a cyclic graph can still be parametrized by a set of $N$ energy parameters $E_{i}, N$ barrier sizes $W_{i}$, and, in addition, by a set of free energy inputs on each clockwise (anti-clockwise) transition, $f_{i}^{+}\left(f_{i}^{-}\right)$, such that

$$
k_{i-1}^{+}=k e^{\beta\left(E_{i-1}-W_{i}+f_{i-1}^{+}\right)}, \quad k_{i}^{-}=k e^{\beta\left(E_{i}-W_{i}+f_{i}^{-}\right)} .
$$

Comparing with Eq. (21), we have the duality relations for the free energy inputs:

$$
f_{i, \mathrm{~B}}^{\mathrm{d}+}(t)=f_{i, \mathrm{~F}}^{-}(\tau-t), \quad f_{i, \mathrm{~B}}^{\mathrm{d}-}(t)=f_{i-1, \mathrm{~F}}^{+}(\tau-t) .
$$

The increased entropy after one clockwise cycle in the dual graph is exactly the decreased entropy after one clockwise cycle in the original one:

$$
\mathcal{A}=\sum_{i} \ln \left(k_{i}^{+} / k_{i}^{-}\right)=-\sum_{i} \ln \left(k_{i}^{\mathrm{d}+} / k_{i}^{\mathrm{d}-}\right) .
$$

The entropy production, $\mathcal{A}$, sometimes is called the macroscopic thermodynamic affinity [1] and cycle force [2]. This reversed entropy production is consistent with the observation that the dual and original model have the inverted energy landscape.

Recently, Sinitsyn et al have shown [21] that for arbitrary closed networks with two driving protocols, such that either

(I) only barrier sizes are driven, or

(II) only node's energies are driven, while keeping all other parameters, including $\mathcal{A}$, constant (which is equivalent in our case to keeping either $\ln \left(k_{i}^{+} / k_{i+1}^{-}\right)$ or $\ln \left(k_{i}^{\mathrm{d}+} / k_{i+1}^{\mathrm{d}-}\right) \equiv \ln \left(k_{i}^{-} / k_{i}^{+}\right)$constant $)$, the following expression relates probabilities of conserved current, $J^{c}$, for forward and backward driving protocols:

$$
\frac{P_{\mathrm{F}}\left(J^{c}\right)}{P_{\mathrm{B}}\left(-J^{c}\right)}=e^{\mathcal{A} J^{c}} .
$$

This Fluctuation Relation for Currents is a generalization of previously known relation for steady state currents [22] to two classes of explicitly time dependent driving protocols. Its proof for graphs of arbitrary geometry in [21] was based on symmetries of the twisted master operator and its supersymmetric counterpart. Duality now allows us to achieve the same result for the cyclic graph with much less efforts. Indeed, the case (I) directly follows from the well known work relation [22, 23]:

$$
\frac{P_{\mathrm{F}}(\mathcal{W})}{P_{\mathrm{B}}(-\mathcal{W})}=e^{\mathcal{W}}
$$

The work $\mathcal{W}$ in our case is defined during a periodic driving cycle, which, in the large time limit $n \rightarrow \infty$ can be written as [24]

$$
\mathcal{W}=\mathcal{A} J^{c}+\int_{0}^{\tau} d t\left[\sum_{i} \dot{E}_{i}(t) \eta_{i}(t)\right],
$$

where $\boldsymbol{\eta}(t)$ is the random process given by $\eta_{i}(t)=1$ if the node $i$ has the particle inside it at time $t$ and otherwise zero.

When only barriers are driven, the second term in Eq. (30) is identically zero, so the work per period is given by $\mathcal{W}=$ $\mathcal{A} J^{c}$, just like in the static case. Since work in case (I) is proportional to $J^{c}$ with a constant factor, $\mathcal{A}$, the relation (28) for $J^{c}$ is consequently satisfied.

The case (II) does not straightforwardly follow from Eq. (30), however, using the previously discussed duality between protocols with only energies and only barriers driven, we should conclude that Eq. (28) should be satisfied for protocols with only energies driven, which concludes its proof. 


\section{DUALITY IN TWISTED FOKKER-PLANCK EQUATION}

Here we will explore the duality relations that are hidden in the twisted Fokker-Planck equations. The additional goal is to obtain the continuous limit of the recently discovered fluctuation relations for currents [21] which so far have been discussed only in the framework of the discrete graph models.

Let us take the jump (transition) spacing $\Delta x$, denote $\chi / \Delta x \rightarrow \chi$ and consider the continuous limit: $\Delta x \rightarrow 0$. The twisted Fokker-Plank equation is thus defined as the continuous limit of the discrete twisted master equation, which reads

$$
\begin{aligned}
\partial_{t} \rho_{\chi}(x, t) & =\hat{L}_{\chi} \rho_{\chi}(x, t), \quad \text { with } \\
\hat{L}_{\chi} & =\left(\chi-\partial_{x}\right)\left[A(x, t)+\left(\chi-\partial_{x}\right) B(x, t)\right],
\end{aligned}
$$

where $\hat{L}_{\chi}$ is the twisted Fokker-Planck operator with auxiliary counting parameter $\chi \cdot \rho_{\chi}(x, t)$ is the generating function of probabilities of a particle being at position $x$ at time $t$ having produced a current $J$ by this time. $A(x, t)=$ $\Delta x\left[k^{+}(x, t)-k^{-}(x, t)\right]$ denotes the drift term and $B(x, t)=$ $\Delta x^{2}\left[k^{+}(x, t)+k^{-}(x, t)\right] / 2$ indicates the diffusion. This twisted Fokker-Planck equation, as the continuous version of the twisted master equation for cyclic graphs, describes the one-dimensional stochastic motion with periodic boundary condition.

Let us define two auxiliary functions [25, 26]: $\psi(x)=$ $-\int_{0}^{x} d y A(y) / B(y)$, and $\varphi(x)=\ln B(x)+\psi(x)$, such that the above equation is characterized equally well by these two functions as by the drift, $A$, and the diffusion, $B$. These two auxiliary functions render us able to recast Eq. (31) as

$$
\partial_{t} \rho_{\chi}(x, t)=\left(\chi-\partial_{x}\right) e^{-\psi}\left(\chi-\partial_{x}\right) e^{\varphi} \rho_{\chi}(x, t) .
$$

Further, by introducing two twisted operators: $\hat{\mathcal{Q}}_{\chi} \equiv\left(\chi-\partial_{x}\right)$ and $\hat{J}_{\chi} \equiv e^{-\psi}\left(\chi-\partial_{x}\right) e^{\varphi}$, we can reconstruct $\hat{L}_{\chi}$, as well as the other three operators as follows,

$$
\begin{aligned}
& \hat{L}_{\chi}=\hat{\mathcal{Q}}_{\chi} \hat{J}_{\chi}=\left(\chi-\partial_{x}\right) e^{-\psi}\left(\chi-\partial_{x}\right) e^{\varphi} ; \\
& \hat{L}_{-\chi}^{\mathrm{d}}=\hat{\mathcal{Q}}_{\chi}^{\mathrm{T}} \hat{J}_{\chi}^{\mathrm{T}}=\left(-\chi-\partial_{x}\right) e^{\varphi}\left(-\chi-\partial_{x}\right) e^{-\psi} ; \\
& \widetilde{L}_{-\chi}=\hat{J}_{\chi}^{\mathrm{T}} \hat{\mathcal{Q}}_{\chi}^{\mathrm{T}}=e^{\varphi}\left(-\chi-\partial_{x}\right) e^{-\psi}\left(-\chi-\partial_{x}\right) ; \\
& \widetilde{L}_{\chi}^{\mathrm{d}}=\hat{J}_{\chi} \hat{\mathcal{Q}}_{\chi}=e^{-\psi}\left(\chi-\partial_{x}\right) e^{\varphi}\left(\chi-\partial_{x}\right) .
\end{aligned}
$$

Following the same arguments as for the discrete master equation, it is straightforward to find that these four operators have the identical eigenvalues as well and share the relations depicted in Fig. 2] A continuous analog of Eq. (18) thus is given by

$$
\varphi^{\mathrm{d}}(x, t)=-\psi(x, t), \quad \psi^{\mathrm{d}}(x, t)=-\varphi(x, t),
$$

The relations (19) and (20) also hold true in the continuum limit, if we define the evolution operators for the twisted Fokker-Planck operators accordingly. We further note that Eq. (35) and (36) are equivalent to $\widetilde{L}_{-\chi}=\left(-\chi+\varphi^{\prime}-\right.$ $\left.\partial_{x}\right) e^{-\psi}\left(-\chi+\varphi^{\prime}-\partial_{x}\right) e^{\varphi}$, and $\widetilde{L}_{\chi}^{\mathrm{d}}=\left(\chi-\psi^{\prime}-\partial_{x}\right) e^{\varphi}(\chi-$ $\left.\psi^{\prime}-\partial_{x}\right) e^{-\psi}$, which lead to

$$
\widetilde{L}_{-\chi}=\hat{L}_{-\chi+\varphi^{\prime}}, \quad \text { and } \quad \widetilde{L}_{\chi}^{\mathrm{d}}=\hat{L}_{\chi-\psi^{\prime}}^{\mathrm{d}},
$$

with the prime meaning the derivative with respect to $x$.

\section{A. Fluctuation Relations for Currents in one dimension}

Duality and symmetry relations on the level of twisted operators allows us to derive various relations between statistics of currents in original and dual models driven by periodic, and otherwise arbitrary, protocols. Considering the isospectral property of these twisted operators, their cumulant generating functions satisfy:

$$
\mu_{-\chi+\varphi^{\prime}, \mathrm{B}}=\mu_{\chi, \mathrm{F}}=\mu_{-\chi, \mathrm{B}}^{\mathrm{d}}=\mu_{\chi-\psi^{\prime}, \mathrm{F}}^{\mathrm{d}},
$$

with the dual relations:

$$
\begin{gathered}
\psi_{\mathrm{F}}^{\mathrm{d}}(t)=-\varphi_{\mathrm{F}}(t)=-\varphi_{\mathrm{B}}(\tau-t)=\psi_{\mathrm{B}}^{\mathrm{d}}(\tau-t), \\
\varphi_{\mathrm{F}}^{\mathrm{d}}(t)=-\psi_{\mathrm{F}}(t)=-\psi_{\mathrm{B}}(\tau-t)=\varphi_{\mathrm{B}}^{\mathrm{d}}(\tau-t) .
\end{gathered}
$$

After the inverse Legendre transformation [10, 15] and under some constraint, we are capable to obtain various fluctuation relations for currents. For instance, the second equality in Eq. (39) leads to the identical distribution of opposite currents generated by original and dual models with timereversed driving protocols:

$$
P_{\mathrm{F}}\left(J^{c}\right)=P_{\mathrm{B}}^{\mathrm{d}}\left(-J^{c}\right),
$$

which is the same as the Eq. (23) obtained for discrete graph. We note that Eq. (41) holds true for arbitrary time-dependent protocols. Now, let us have either time-independent $\varphi^{\prime}(x)$ or time-independent $\psi^{\prime}(x)$, which is similar to the two required protocols for obtaining Eq. (28) in the discrete version. In this way, the first or the last equality in Eq. (39) provides the relations for current distributions generated by forward and backward protocols in the original and dual models, respectively:

$$
\frac{P_{\mathrm{F}}\left(J^{c}\right)}{P_{\mathrm{B}}\left(-J^{c}\right)}=e^{\mathcal{A} J^{c}} ; \quad \frac{P_{\mathrm{F}}^{\mathrm{d}}\left(J^{c}\right)}{P_{\mathrm{B}}^{\mathrm{d}}\left(-J^{c}\right)}=e^{-\mathcal{A} J^{c}} .
$$

Here, the constant, $\mathcal{A}$, which is defined as $\oint d x A(x, t) / B(x, t)$, is also given by

$$
\mathcal{A}=-\oint d x \varphi^{\prime}(x, t)=-\oint d x \psi^{\prime}(x, t)
$$

which is similar to its discrete version (27). In fact, as $\left|k^{+}(x, t)-k^{-}(x, t)\right| \ll k^{+}(x, t), k^{-}(x, t)$,

$$
\frac{A(x, t)}{B(x, t)} \Delta x=\frac{2\left[k^{+}(x, t)-k^{-}(x, t)\right]}{k^{+}(x, t)+k^{-}(x, t)} \approx \ln \frac{k^{+}(x, t)}{k^{-}(x, t)},
$$

so that, after a closed cycle,

$$
\oint \frac{A(x, t)}{B(x, t)} d x \Leftrightarrow \sum_{i} \ln \frac{k_{i}^{+}(t)}{k_{i}^{-}(t)} .
$$


It is clear that only the nonconservative part of $A(x, t) / B(x, t)$ contributes to $\mathcal{A}$. It plays the role of nonequilibrium source, which is related to the macroscopic thermodynamic affinity [1] and cycle force [2]. If $\mathcal{A}$ is zero, the system is at the situation that no directed transport occurs at the steady state [27].

\section{B. No-Go Theorems for One-Dimensional Brownian Motor}

The duality relations, which are hidden in the twisted Fokker-Planck equations, shed a new light on some of the previously known no-pumping (no-go) theorems for a one-dimension Brownian motor [26]. If $\varphi(x)$ holds timeindependent, the system has the steady state solution: $\rho(x) \sim$ $e^{-\varphi(x)}$ so that the average current vanishes, no matter how arbitrarily we drive $\psi(x, t)$. Therefore, the dual relation between $\varphi$ and $\psi$ offers us the no-pumping theorem stating that a current on average can not be pumped, also in case when $\psi(x)$ is fixed, which is the same as to saying that there is no-pumping situation when the ratio $A(x, t) / B(x, t)$ is time-independent [26]. One concrete example is the overdamped Brownian motor with non-constant friction coefficient: $\partial_{t} \rho(x, t)=\partial_{x}\left(U^{\prime}(x) / \gamma(x, t) \rho(x, t)\right)+$ $\partial_{x}^{2}\left(k_{B} T / \gamma(x, t) \rho(x, t)\right)$. No matter how we vary the friction coefficient $\gamma(x, t)$, the constant $\psi(x)=-\int_{0}^{x} d y U^{\prime}(y) / k_{B} T$ guarantees the result that the average current is zero, which is consistent with the previously found no-go theorem [28].

Actually, under the detailed balance condition, we can write $k^{+} \sim e^{E(x, t)-W(x+\Delta x, t)}$ and $k^{-} \sim e^{E(x, t)-W(x-\Delta x, t)}$. Following these expressions, it is straightforward to show that $A \sim-W^{\prime} e^{E-W}$ and $B \sim e^{E-W}$, so that $\psi \sim W, \varphi \sim E$. Thus, the duality between $\varphi$ and $\psi$ is similar to the duality between energy wells and barriers in discrete graphs. Considering the energy landscape between original and dual model is reverted, the signs before $\mathcal{A}$ in fluctuation relations Eq. (42) are opposite.

If after inverting the energy landscape or exchanging the energy wells and barriers, a model is invariant, we say this model is self-dual. In this case, the relation (41) changes to
$P_{\mathrm{F}}\left(J^{c}\right)=P_{\mathrm{B}}\left(-J^{c}\right)$. Once additionally, the driving protocol follows the time inversion invariance, i.e., $\mathrm{B}=\mathrm{F}$, we have $P_{\mathrm{F}}\left(J^{c}\right)=P_{\mathrm{F}}\left(-J^{c}\right)$, which leads to the no-pumping $\left\langle J^{c}\right\rangle=0$ naturally. In fact, this self-duality, combined with the time inversion invariance, recovers the supersymmetry criterion for vanishing currents in Brownian motors [28]: $-V(x, f(t))=V(x+L / 2, f(-t))$, where the driving has time-reversal symmetry: $f(t)=f(-t)$ and the potential is self-dual: $-V(x)=V(x+L / 2)$ with $L / 2$ determined by its periodicity $L$. Our relation $P_{\mathrm{F}}\left(J^{c}\right)=P_{\mathrm{F}}\left(-J^{c}\right)$ offers more information for this case because it implies that not only the average current but also all the odd cumulants of the current are zero.

\section{SUMMARY}

Dualities have proven to be a fruitful guide to many fundamental problems in physics. Our duality relations for stochastic currents show that fluctuation theorems for currents exist beyond standard fluctuation theorems for work and entropy production. Existence of duality relation also provides new means to search for new exact results in mesoscopic statistical mechanics because some of already known exact results may have their dual counterparts, as for the case of no-pumping theorems. We hope our results will shed new light on the study of time dependent nonequilibrium transport, provide new insights to understanding working mechanisms of biological molecular motors, and will be applied to optimal design of various artificial molecular motors [29] and nanoscale pumps.

Acknowledgment. J.R. thanks the support of Baowen Li and hospitality of Jian-Xin Zhu during his visit to LANL. N.A.S. thanks Jordan Horowitz for useful discussion at early stages of this research. The work at LANL was carried out under the auspices of the National Nuclear Security Administration of the U.S. Department of Energy at Los Alamos National Laboratory under Contract No. DE-AC52-06NA25396. It is also based upon work supported in part by the National Science Foundation under CHE-0808910 at WSU, and under ECCS0925618 at NMC.
[1] J. Schnakenberg, Rev. Mod. Phys. 48, 571 (1976).

[2] T. L. Hill, Free Energy Transduction and Biochemical Cycle Kinetics (Springer-Verlag, New York, 1989).

[3] D. R. Astumian, Annu. Rev. Biophys. 40, 289313 (2011).

[4] B. Derrida and J. L. Lebowitz, Phys. Rev. Lett. 80, 209 (1998).

[5] N. A. Sinitsyn and I. Nemenman, Europhys. Lett. 7758001 (2007); V. Y. Chernyak and N. A. Sinitsyn, J. Chem. Phys. 131, 181101 (2009).

[6] D. Astumian, Proc. Natl. Acad. Sci. U.S.A. 104, 19715 (2007).

[7] S. Kohler, J. Lehmann, and P. Hänggi, Phys. Rep. 406, 379 (2005).

[8] D. A. Bagrets and Y. V. Nazarov, Phys. Rev. B 67, 085316 (2003).

[9] J. Ren, P. Hänggi, and B. Li, Phys. Rev. Lett. 104, 170601 (2010); J. Ren and B. Li, Phys. Rev. E 81, 021111 (2010).
[10] M. Esposito, U. Harbola, and S. Mukamel, Rev. Mod. Phys. 81, 1665 (2009).

[11] R. L. Jack and P. Sollich, J. Stat. Mech. P11011 (2009).

[12] A. Dembo and O. Zeitouni, Large Deviations: Techniques and Applications, 2nd ed. (Springer, 1998).

[13] H. Touchette, Phys. Rep. 478, 1 (2009).

[14] N. A. Sinitsyn, J. Phys. A: Math. Theor. 42, 193001 (2009).

[15] D. Andrieux and P. Gaspard, J. Stat. Phys. 127, 107 (2007).

[16] I.V. Gopich and A. Szabo, J. Chem. Phys. 124, 154712 (2006).

[17] J. Tailleur, J. Kurchan, and V. Lecomte, J. Phys. A: Math. Theor. 41, 505001, (2008).

[18] S. Rahav, J. Horowitz and C. Jarzynski, Phys. Rev. Lett. 101, 140602 (2008).

[19] V. Y. Chernyak and N. A. Sinitsyn, Phys. Rev. Lett. 101, 160601 (2008). 
[20] C. Maes, K. Netocny, S. R. Thomas, J. Chem. Phys. 132, 234116 (2010).

[21] N. A. Sinitsyn, A. Akimov, and V. Y. Chernyak, Phys. Rev. E 83, 021107 (2011).

[22] G. N. Bochkov and Yu. E. Kuzovlev, Sov. Phys. JETP 49543 (1979); Physica A 106, 443 (1981).

[23] G. E. Crooks, J. Stat. Phys. 90, 1481 (1998).

[24] E. Boksenbojm, B. Wynants, and C. Jarzynski, "Lecture notes. Fundamental Problems in Statistical Physics", XII, Leuven,
Preprint: arXiv/1002.1230, (2009).

[25] H. Risken, The Fokker-Planck Equation: Methods of Solution and Applications (Springer, New York, 1984).

[26] J. E. Horowitz and C. Jarzynski, J. Stat. Phys. 136, 917 (2009). [27] H. Qian, Phys. Rev. Lett. 81, 3063 (1998).

[28] P. Reimann, Phys. Rep. 361, 57 (2002).

[29] P. Hänggi and F. Marchesoni, Rev. Mod. Phys. 81, 387 (2009). 